\title{
ARTÍCULO DE REVISIÓN \\ Síndrome coronario agudo atípico, un reto diagnóstico: revisión de tema
}

Fecha de recibido:

6 de octubre de 2020 .

Fecha de aprobación: 13 de enero de 2021.
Forma de citar este artículo: Hoyos S, Botero DA, Agudelo I, Ortiz A, Arango JJ, Díaz JJ, et al. Síndrome coronario agudo atípico, un reto diagnóstico: revisión de tema. Med UPB. 2021;40(2):-

DOI:10.18566/medupb.v40n2.a08

1 Facultad de Medicina, Universidad CES. Medellín, Colombia

2 División de Investigación e innovación, Facultad de Medicina, Grupo de Investigación Especialidades MédicoQuirúrgicas, Universidad CES. Medellín, Colombia

3 Servicio de Cardiología, Clínica CardioVID.

Medellín, Colombia

Dirección de

correspondencia: Sebastián Hoyos Gutiérrez. Correo electrónico: shoyosg@ outlook.com

\author{
A review on Atypical Acute Coronary Syndrome, a diagnostic \\ challenge / Síndrome Coronariana Aguda Atípica, Um Desafio \\ Diagnóstico: Revisão De Tópico
}

Sebastián Hoyos Gutiérrez ${ }^{1}$, Diego Alejandro Botero López ${ }^{1}$, Isabela Agudelo Aguilar ${ }^{1}$, Alejandra Ortiz Moreno ${ }^{1}$, Juan José Arango Serrano ${ }^{1}$, Juan José Díaz Gamboa ${ }^{1}$, Nelcy Lorena Valencia Ortiz², Carlos Arturo Martínez Cano ${ }^{3}$

\section{RESUMEN}

El síndrome coronario agudo (SCA) es la principal causa de muerte, por esta razón, es fundamental reconocer sus características clínicas. Tradicionalmente ha sido descrito un cuadro denominado típico, consistente en dolor torácico retroesternal o en la región izquierda del tórax, explicado como una sensación de presión o pesadez, de duración superior a 20 minutos, que se puede irradiar a la extremidad superior izquierda o derecha, cuello o mandíbula, asociado a diaforesis y náuseas. Diversos grupos de pacientes como mujeres, diabéticos, ancianos y con antecedentes de falla cardiaca o accidente cerebrovascular presentan cuadros llamados atípicos, que en gran proporción no manifiestan dolor torácico. Varios estudios plantean que no hay síntomas suficientemente sensibles y específicos para ser considerados típicos en el contexto del SCA, por lo que el diagnóstico debe incluir además de la evaluación clínica, el electrocardiograma, los factores de riesgo y los biomarcadores. El uso de puntajes de riesgo como el HEART ha demostrado ser útil en este escenario.

Palabras clave: síndrome coronario agudo; manifestaciones clínicas; equivalentes anginosos; angina atípica

\section{ABSTRACT}

Acute coronary syndrome (ACS) is the main cause of mortality around the world; therefore, it is essential to recognize the clinical characteristics that increase its diagnostic suspicion. Traditionally, it has been defined as a so-called typical condition, consisting of retrosternal chest pain or pain in the left region of the thorax. It is described as a feeling of pressure or heaviness, lasting more than 20 minutes, which can radiate to the left or right upper limb, neck or jaw, and can be associated with diaphoresis and nausea. Various groups of patients such as women, diabetics, the elderly, and those with a history of heart failure or cerebrovascular accident, have so-called atypical clinical sets of symptoms, which in a large proportion may not present chest pain. Various studies suggest that there are not sufficiently sensitive and specific symptoms to be considered typical in the context of ACS, thus, the approach must include the electrocardiogram, risk factors and biomarkers in addition to the clinical evaluation. The use of risk scores such as the HEART has proven very useful in this scenario.

Keywords: acute coronary syndrome; clinical presentation; angina equivalents; atypical angina

\section{RESUMO}

A síndrome coronariana aguda (SCA) é a principal causa de morte, por isso é fundamental o reconhecimento de suas características clínicas. Tradicionalmente, é descrita uma condição dita típica, que consiste em dor torácica retroesternal ou na região esquerda 
do tórax, explicada como uma sensação de pressão ou peso, com duração superior a 20 minutos, que pode irradiar para a esquerda ou direita membro superior, pescoço ou mandíbula, associado a sudorese e náusea. Vários grupos de pacientes como mulheres, diabéticos, idosos e com história de insuficiência cardíaca ou acidente vascular cerebral apresentam condições atípicas, que em grande parte não manifestam dor no peito. Vários estudos sugerem que não existem sintomas suficientemente sensíveis e específicos para serem considerados típicos no contexto da SCA, de modo que o diagnóstico deve incluir, além da avaliação clínica, o eletrocardiograma, fatores de risco e biomarcadores. $\mathrm{O}$ uso de escores de risco, como o CORAÇÃO, tem se mostrado útil nesse cenário.

Palavras-chave: síndrome coronariana aguda; manifestações clínicas; equivalentes anginosos; angina atípica

\section{INTRODUCCIÓN}

E1 SCA hace referencia al espectro de presentaciones agudas de la enfermedad coronaria, que requieren una valoración de emergencia, dado su alto potencial de morbilidad y mortalidad ${ }^{1}$. Dentro del espectro de presentación se encuentran el infarto agudo de miocardio con elevación del ST (IAMCEST), el infarto agudo de miocardio sin elevación del ST (IAMSEST) y la angina inestable $(\mathrm{AI})^{2}$.

La importancia de reconocer el SCA radica en que, según la Organización Mundial de la Salud (OMS), la cardiopatía isquémica es la principal causa de defunción ${ }^{3}$. En Colombia, según el Departamento Administrativo Nacional de Estadísticas (DANE), las enfermedades isquémicas del corazón fueron la principal causa de defunción para el año $2018^{4}$.

Las características del SCA han sido descritas desde épocas remotas. En 1768, William Heberden presentó una de las descripciones más antiguas de la angina pectoris ${ }^{5} \mathrm{y}$, gracias a múltiples estudios observacionales posteriores, se ha logrado una caracterización precisa del espectro de presentación de la enfermedad coronaria, que permite saber que no todos los pacientes con SCA llegan al servicio de urgencias manifestando un cuadro típico, lo que llega a generar confusión en los médicos y retrasa el diagnóstico y tratamiento oportunos ${ }^{6,7}$.

La presentación clínica que tradicionalmente ha sido descrita como típica, consiste en un dolor similar al de la angina de esfuerzo estable, más intenso y duradero, que se presenta en el área retroesternal, con o sin extensión al lado izquierdo del tórax, descrito por el paciente como una sensación de presión o pesadez, de duración superior a 20 minutos, que se puede irradiar a la extremidad superior izquierda o derecha, cuello o mandíbula y puede estar asociada a diaforesis, náuseas, dolor abdominal, síncope o disnea ${ }^{8-10}$. Con menos frecuencia, los pacientes exponen dolor en el hemitórax derecho. El signo de Levine, en el que el paciente pone su puño cerrado sobre el pecho al describir su dolor, es visto comúnmente en pacientes con infarto agudo de miocardio ${ }^{11}$.

Por el contrario, la presentación atípica se manifiesta como molestia epigástrica o síntomas diversos y menos específicos, incluso sin malestar torácico ${ }^{12}$. En este artículo se revisan las manifestaciones atípicas del SCA, dado que hasta un $33 \%$ de los pacientes no tiene el cuadro típico al momento de consultar ${ }^{13}$.

\section{TEMA CENTRAL}

Las manifestaciones clínicas denominadas atípicas son descritas por diversos autores como cuadros de disnea, palpitaciones, diaforesis, náuseas/vómitos o debilidad, sin dolor torácico, llamados equivalentes anginosos ${ }^{13,14,15} \mathrm{o}$, según Greenslade et al., como un dolor limitado al epigastrio o dorsal, de características quemantes, punzantes o típico de la indigestión ${ }^{16}$. Se han documentado subgrupos de pacientes más propensos a las presentaciones atípicas, que son un reto diagnóstico que retrasan la realización de intervenciones terapéuticas oportunas ${ }^{13}$.

En un estudio multicéntrico de pacientes con infarto agudo de miocardio (IAM), el 33\% presentó manifestaciones atípicas o sin dolor torácico. Este grupo tenía un promedio de edad mayor que los pacientes con dolor torácico (74.2 versus 66.9 años), mayor proporción de mujeres (49\% versus $38 \%$ ) y pacientes diabéticos $(32.6 \%$ versus $25.4 \%$ ) o con falla cardiaca previa (26.4\% versus 12.3\%). También se encontró que de los pacientes que tuvieron antecedente de enfermedad cerebrovascular, un $47 \%$ tuvo manifestaciones atípicas. En aquellos con presentación atípica se documentó un mayor tiempo para consultar al hospital (7.9 versus 5.3 horas), además tuvieron menor probabilidad de diagnóstico de SCA al momento de la admisión (22.2\% versus 50.3\%), lo que redujo la posibilidad de un tratamiento oportuno ${ }^{13}$.

Estos datos son coherentes con una revisión de tema 
realizada en Bogotá (Colombia), donde se especifica que del 5\% al 10\% de los pacientes con IAM es dado de alta debido a errores en el diagnóstico, con mortalidad del $6 \%$ al $8 \%{ }^{17}$.

Entre los factores de riesgo para IAM sin dolor torácico están el antecedente de falla cardiaca, con un OR de 1.77; el accidente cerebrovascular previo, con un OR de 1.43, la edad mayor a 65 años, con un OR de 1.28; la presencia de diabetes mellitus, con un OR de 1.21; el género femenino, con un OR de 1.06 y la raza distinta a la blanca, con un OR de 105. A la inversa, solo un $17.5 \%$ de los pacientes que no tienen los citados antecedentes presenta un IAM sin dolor torácico. Con solo presentar tres de los anteriores antecedentes se aumenta la probabilidad de un IAM sin dolor en un 50\%. Adicionalmente, otros contextos clínicos también se relacionan con el IAM sin dolor torácico, entre estos se encuentran ser fumador, con un OR de 0.87; el antecedente de cirugía de bypass coronario, con un OR de 0.82; el antecedente familiar de enfermedad arterial coronaria, con un OR de 0.74; el antecedente de hipercolesterolemia, con un OR de 0.77; la historia de angioplastia coronaria, con un OR de 0.73; y la presencia de angina previa, con un OR de $0.69^{13}$.

A continuación, se detallan los grupos que presentan manifestaciones atípicas con mayor frecuencia:

\section{Mujeres}

Se ha evidenciado que las manifestaciones del SCA pueden variar entre ambos sexos, son las mujeres quienes tienen más frecuentemente presentaciones atípicas. Esto porque ellas tienen predominio de SCA no obstructivo, con erosiones parciales de placas ateroescleróticas, que causan estenosis menos graves y generan trombos más organizados, que permanecen por mayor tiempo (hasta siete días) antes de provocar síntomas, favoreciendo la embolización distal con oclusión microvascular. Por el contrario, se ha encontrado que la ruptura total o subtotal de la placa ateroesclerótica es más común en los hombres, es más grave y con mayor riesgo de oclusión total ${ }^{18}$.

Estudios previos demuestran que las mujeres usan con mayor frecuencia los servicios de salud, sin embargo, tienen menor acceso a las pruebas diagnósticas y al tratamiento para el SCA, también tardan más en consultar (tres horas) ${ }^{18,19}$. Una de las razones es que, si bien ambos géneros pueden presentar las mismas manifestaciones clínicas de SCA, hay una diferencia en la proporcionalidad de la sintomatología, porque es posible que en las mujeres el dolor torácico no sea el primer síntoma o el más significativo desde la percepción subjetiva, por lo que no es suficiente para buscar atención médica oportuna ${ }^{19}$. Se deben tener en cuenta aspectos fisiopatológicos adicionales, como: los efectos hormonales sobre los marcadores inflamatorios, la distribución del tejido graso y la carga aterosclerótica, así como la isquemia y disfunción vascular inducida por el estrés mental, que ocurre con más frecuencia en las mujeres ${ }^{19}$.

La revisión sistemática del grupo de Patel registró que el dolor torácico fue el síntoma más reportado por hombres y mujeres. Para el sexo femenino fue transitorio con mayor frecuencia y de características agudas o de tipo punzante, no hubo diferencias significativas en cuanto al dolor de extremidades superiores y de hombros, así como tampoco en síntomas como dolor epigástrico, diaforesis, mareo y síncope. Sin embargo, se evidenció que las mujeres experimentaron más disnea, palpitaciones, fatiga, náuseas y vómitos, que fueron considerados como síntomas de alarma iniciales ${ }^{19}$.

Un estudio prospectivo evidenció que la opresión en el pecho es un síntoma sensible ( $63 \%$ en hombres versus $66 \%$ en mujeres) pero inespecífico ( $41 \%$ en hombres versus $36 \%$ en mujeres). Del mismo modo, el dolor torácico fue sensible ( $72 \%$ en hombres versus $67 \%$ en mujeres) pero la especificidad fue baja (37\% en hombres versus $36 \%$ en mujeres). No hay datos suficientes para hablar de una presentación atípica en mujeres, puesto que esto implica tener una sintomatología estándar y hay pocos síntomas sensibles o específicos para el diagnóstico del SCA. De hacer referencia entonces a una presentación atípica debería proveerse el grupo de referencia al que aplican los términos (por ejemplo, hombres versus mujeres), pues se han encontrado síntomas con mayor sensibilidad en las mujeres, como: indigestión (30\% versus $18 \%$ ), disnea ( $58 \%$ versus $41 \%$ ), palpitaciones ( $27 \%$ versus $17 \%$ ) y dolor del dorso superior (34\% versus $14 \%)^{21}$.

\section{Diabetes}

Hasta un $40 \%$ de los pacientes que presentan SCA tiene diabetes mellitus (DM). Los diabéticos tienen un riesgo de dos a cuatro veces mayor de desarrollar enfermedad arterial coronaria y, por tanto, mayor riesgo de presentar un SCA. Una vez lo padecen, tienen mayor mortalidad (2-3 veces). Adicionalmente, al ser hospitalizados tienen mayor probabilidad de complicaciones como falla cardiaca, eventos cerebrovasculares, sangrados, falla renal e IAM recurrente ${ }^{22}$.

Debido a la neuropatía sensorial y autonómica, los diabéticos tienen también mayor posibilidad de síntomas atípicos de SCA. Una angina pudiese no ser identificada de manera oportuna, lo que retrasa el momento de la consulta y el de inicio del tratamiento, con el consecuente mal pronostico ${ }^{12}$. Un estudio que incluyó 16115 pacientes hospitalizados con SCA, encontró que los que tenían DM eran de edades más avanzadas, en su mayoría eran mujeres y tenían una prevalencia más elevada de otras 
comorbilidades ${ }^{23}$, lo que indica que hay mayor proporción de otras condiciones que predisponen a manifestaciones atípicas del SCA.

Según el estudio de Coronado et al., de 61 pacientes con SCA asociado a DM, 35 presentaron SCA sin dolor torácico y 26 fueron diagnosticados con SCA con dolor torácico $(\mathrm{p}=0.01)$. Además, los síntomas más comunes en diabéticos con SCA son en su mayoría inespecíficos e incluyen disnea, náusea, vómitos, diaforesis, o fatiga inexplicada ${ }^{12}$.

En un estudio realizado de DeVon et al., en 256 pacientes hospitalizados con diagnóstico de SCA, se midió la intensidad de los síntomas al momento de la admisión al hospital y los pacientes diabéticos de larga data (mayor a 10 años desde el diagnóstico) reportaban mayor gravedad de fatiga, hiperventilación, y dificultad respiratoria. En los pacientes con DM de menos de 10 años de duración, no se encontró mayor intensidad de la fatiga ni de la hiperventilación en comparación con los no diabéticos, lo que está en relación con los cambios microvasculares y la disfunción autonómica que ocurren con el tiempo en la $\mathrm{DM}^{24}$.

Datos del estudio BARI 2-D, en pacientes con DM Tipo II que fueron revascularizados, permite constatar que de 2319 casos analizados, el 19\% manifestó angina típica, el $42 \%$ describió angina típica más síntomas equivalentes anginosos asociados, el $21 \%$ presentó únicamente equivalentes anginosos y el 18\% era asintomático. Los hombres mayores de 60 años, los que realizaban actividad física y quienes tomaban tiazolidinedionas tuvieron un OR inferior a la unidad para angina típica. Por el contrario, los pacientes revascularizados previamente tuvieron cuadros típicos con mayor frecuencia ${ }^{25}$.

Otra salvedad a la hora de enfocar un SCA en el diabético, es el efecto de los hipoglicemiantes orales sobre los cambios electrocardiográficos (ECG). Por ejemplo, en modelos animales, se ha encontrado que el uso de sulfonilureas disminuye la elevación del ST y la elevación y forma picuda de la onda T. Así pues, en estos pacientes se debe mantener un alto índice de sospecha y realizar una evaluación cuidadosa en el servicio de urgencias ${ }^{22,26}$.

\section{Ancianos}

E1 SCA representa la principal causa de muerte en personas mayores de 65 años. En aquellos que sobreviven suelen haber importantes alteraciones de la calidad de vida. A pesar de su relevancia, el abordaje inicial es usualmente errado y comúnmente se realiza un tratamiento inadecuado ${ }^{27}$. En el registro GRACE se evidenció la relación entre la edad y la presentación de IAM sin elevación del ST, que llega hasta el $41 \%$ en mayores de 85 años, frente a un 30\% en menores de 65 años, el IA-
MCEST fue más común en jóvenes que en ancianos ${ }^{28}$. Otro aspecto que considerar es el retraso en la consulta a urgencias, donde un $56.2 \%$ de los mayores de 65 años se demoró entre dos horas y dos días en consultar. Esto es explicado en parte por la ambigüedad de los síntomas y las dificultades cognitivas, físicas y de redes de apoyo ${ }^{27}$.

Con el envejecimiento ocurren cambios que influyen en las manifestaciones clínicas y el manejo de estos pacientes. Dentro de estos cambios se encuentran la mengua de la elasticidad arterial, el aumento de la poscarga, la disfunción diastólica, la disfunción endotelial, y la presencia de un estado proinflamatorio con tendencia a la coagulación. De igual manera, hay cambios como el compromiso de las funciones cognitivas y físicas, la presencia de múltiples comorbilidades y las alteraciones en el metabolismo y la excreción de los fármacos, que son variables para tener en cuenta, debido a la relevancia que tienen en el abordaje diagnóstico y terapéutico ${ }^{20,29}$.

Una de las principales características de los pacientes de edad avanzada, es la presencia de equivalentes anginosos, entendidos como la no presentación de dolor torácico como síntoma principal. En el estudio GRACE, el promedio de edad de los pacientes que se presentaron sin dolor torácico fue de 72.9 años. En el estudio NRMI se evidenció que mientras mayor sea la edad, mayor es la posibilidad de presentaciones atípicas. De hecho, en mayores a 85 años, solo el $40 \%$ manifestó dolor torácico ${ }^{29}$.

Las principales expresiones en pacientes sin dolor torácico fueron: disnea (cuya prevalencia varía entre 49\% y $72 \%$ en diferentes estudios), diaforesis (26\%), náuseas y vómito $(24 \%)^{29}$. Sin embargo, en registros generales de SCA en ancianos, el dolor torácico sigue siendo la principal manifestación. Los pacientes que no presentaron dolor al momento de la consulta tenían mayor número de comorbilidades, siendo una de las más importantes la diabetes, hasta en un 35\% de ellos ${ }^{12}$.

Como consecuencia de la falta de sospecha clínica, por el elevado número de presentaciones atípicas, se ha registrado que los infartos no reconocidos ascienden hasta un $60 \%$ en pacientes mayores de 85 años $^{29}$. Aunque para algunos autores, aún no existan razones establecidas para relacionar la edad con IAM no reconocidos, se ha evidenciado que los cambios cognitivos, las comorbilidades y la disminución de la función sensorial de los nervios con la edad, explican la variabilidad clínica ${ }^{30}$.

La ausencia de dolor torácico, como síntoma principal en edades avanzadas, tiene una relación directa con el aumento de la mortalidad. En mujeres ancianas sin dolor torácico, la mortalidad intrahospitalaria aumenta seis veces respecto a la de mujeres jóvenes con dolor torácico (21.2\% versus $3.7 \%)$, y los hombres ancianos sin dolor torácico presentaron una mortalidad de $22 \%$ respecto de hombres jóvenes con dolor torácico $(2.4 \%)^{27,30}$. 


\section{Insuficiencia cardiaca}

La insuficiencia cardiaca (IC) es un predictor de SCA sin dolor torácico. Se ha visto que la IC más que predisponer a los pacientes a isquemia indolora, provoca disnea y otros signos de agudización de la falla cardiaca, cuando la perfusión miocárdica está gravemente comprometida. La manifestación más frecuente en caso de isquemia indolora en pacientes con IC es la disnea (72\%) asociada a edema pulmonar y acompañada de fatiga extrema, náuseas o debilidad ${ }^{31}$.

Hasta un $40 \%$ de los pacientes con signos y síntomas de IC aguda tienen un SCA como desencadenante ${ }^{32}$. En estos casos el diagnóstico es difícil ya que ambas patologías elevan las troponinas ${ }^{33}$.

El SCA descompensa la IC y los pacientes tienen dolor torácico, ECG diagnóstico o no diagnóstico, pero troponinas cardiacas elevadas. $\mathrm{O}$ bien IC sin dolor torácico, ECG no diagnóstico y troponinas cardiacas elevadas, donde habrá que tener en cuenta que la causa de esta elevación, si bien puede ser la SCA, podría ser la injuria cardiaca que se da en la $\mathrm{IC}^{34}$.

En el estudio PRIMA, para caracterizar la IC en el contexto del SCA, se incluyeron 2507 pacientes, de los que el 33\% presentaba IC Killip II-IV en el momento de la admisión, y se concluyó que la mortalidad hospitalaria incrementó dramáticamente de acuerdo con la clasificación de Killip (Killip I 9\% versus Killip IV 62\%). Además, se encontró que la edad (OR 1.5) y la IC (OR 4.6) son factores de riesgo independientes para presentación atípica de $\mathrm{SCA}$, los que a su vez tienen que ver con la diabetes (OR 1.5), la hipertensión (OR 1.1) y el infarto previo (OR 1.4) ${ }^{31}$.

\section{Accidente cerebrovascular}

Hay factores de riesgo modificables y no modificables, que influyen sobre la presentación del accidente cerebrovascular $(\mathrm{ACV})$ hemorrágico o isquemico ${ }^{35}$. El ACV está entre las condiciones que implican mal pronóstico del $\mathrm{SCA}$, como la disfunción ventricular izquierda grave, la enfermedad renal crónica o la edad avanzada ${ }^{36}$.

E1 estudio CRUSADE revisó en una cohorte de 43239 pacientes, mayores de 65 años, la mortalidad tras un IAMSEST. Alrededor de la mitad de eran mujeres, con una edad media de 77 años con comorbilidades, y eventos previos como ACV (HR 1.31) y otros eventos cardiovasculares $^{37}$.

La historia de ACV es un factor de riesgo de SCA y para la presentación atípica. Se han detectado siete factores clave para la asociación de SCA y ACV: tabaquismo, inactividad física, mala nutrición, obesidad, hipercolesterolemia, diabetes e hipertensión arterial ${ }^{38}$.

\section{Cómo enfocar el paciente con manifestaciones atípicas de SCA}

Debido a que ningún síntoma por sí mismo puede confirmar o descartar el SCA, todo paciente con una manifestación atípica que haga sospechar SCA debe tener un enfoque diagnóstico similar que aquel que presenta síntomas típicos, esto incluye valorar factores de riesgo, cambios electrocardiográficos y las troponinas ${ }^{39}$.

Los modelos de predicción de riesgo cuantitativos, al englobar todas las anteriores consideraciones, han demostrado utilidad diagnóstica y pronóstica. Los puntajes más ampliamente utilizados son el TIMI y el HEART. Al compararlos, se constata que el puntaje HEART tiene mejor desempeño que el TIMI ${ }^{40}$. El HEART fue derivado de las principales características que hacen sospechar un SCA, y supera algunas desventajas del TIMI al puntuar las diferentes particularidades, según nivel de riesgo y no simplemente de manera binaria. Además, incorporó el importante aspecto de la historia clínica, al considerarla desde poco sospechosa (ejemplo: pacientes con manifestaciones atípicas) hasta altamente sospechosa y es útil aun en pacientes de bajo riesgo ${ }^{41}$. Validaciones posteriores han permitido confirmar que puntajes bajos del HEART (0-3) logran excluir eventos adversos mayores a corto plazo y puntajes altos (7-10) se asocian con alto riesgo de eventos adversos mayores, que requieren manejo más agresivo ${ }^{42}$.

\section{CONCLUSIONES}

Ya que el SCA es importante fuente de morbimortalidad, es fundamental tener un claro conocimiento acerca de su diversas presentaciones clínicas. Aunque por lo general se ha descrito un cuadro típico, diversos estudios han demostrado que esta presentación no llega al 70\%, por lo que los autores consideran que el SCA no debe ser diferenciado en típico o atípico, sino que más bien hay que considerar que el SCA tiene una amplia variedad de síntomas y que la ausencia de dolor torácico no excluye el diagnóstico. Existen condiciones como la edad avanzada, el sexo femenino, la diabetes o la IC, que hacen más probable un espectro de presentación atípico y, por tanto, se debe tener un alto índice de sospecha para evitar retrasos en el diagnóstico y en el tratamiento. Los puntajes de riesgo para pacientes con sospecha de síndrome coronario agudo, como el HEART, ayudan a superar dificultades en el diagnóstico.

\section{Declaración de conflictos de intereses}

Los autores declaran no tener ningún conflicto de interés. 


\section{REFERENCIAS}

1. Anderson JL, Morrow DA. Acute myocardial infarction. N Engl J Med 2017;376:2053-64.

2. Amsterdam EA, Wenger NK, Brindis RG, Casey DE, Ganiats TG, Holmes DR, et al. 2014 AHA/ACC Guideline for the management of patients with non-ST-elevation acute coronary syndromes: A report of the American College of Cardiology/American Heart Association Task Force on practice guidelines. Circulation 2014;130:e344-426.

3. OMS. Las 10 principales causas de defunción [Internet]. [Consultado 28 de junio de 2020]. Disponible en: https://www.who.int/es/news-room/fact-sheets/detail/the-top-10-causes-ofdeath

4. DANE. Defunciones no fetales [Internet]. [Consultado 28 de junio de 2020]. Disponible en: https://www.dane.gov.co/index.php/estadisticas-por-tema/salud/nacimientos-y-defunciones/ defunciones-no-fetales

5. Silverman ME. William heberden and some account of a disorder of the breast. Clin Cardiol 1987;10:211-3.

6. Li PWC, Yu DSF. Recognition of atypical symptoms of acute myocardial infarction: Development and validation of a risk scoring system. J Cardiovasc Nurs 2017;32:99-106.

7. Brown JE. Chest Pain. En: Rosen's Emeregency Medicine. 9.a ed. Elsevier; 2018. p. 204-12.

8. Roffi M, Patrono C, Collet J-P, Mueller C, Valgimigli M, Andreotti F, et al. 2015 ESC Guidelines for the management of acute coronary syndromes in patients presenting without persistent ST-segment elevation: Task Force for the Management of Acute Coronary Syndromes in Patients Presenting without Persistent ST-Segment Elevation of the European Society of Cardiology (ESC). Eur Heart J 2016;37:267-315.

9. Scirica BM, Libby P, Morrow DA. Infarto de miocardio con elevación del segmento ST: fisiopatología y evolución clínica. En: Zipes DP MD, Libby P MD, Bonow RO, Mann DL, Tomaselli GF, Braunwald E, editores. Braunwald Tratado de cardiología 2019. p. 1095-122.

10. Diercks DB, Hollander JE, Chang AM. Acute coronary syndromes. En: Tintinalli JE, Ma OJ, Yealy DM, Meckler GD, Stapczynski JS, Cline DM, et al., editores. Tintinalli's Emergency Medicine: A Comprehensive Study Guide, 9e [Internet]. New York, NY: McGraw-Hill Education; 2020 [Consultado 26 de septiembre de 2020]. Disponible en: accessmedicine.mhmedical.com/content. aspx?aid $=1174603293$

11. Theroux P. Acute coronary syndromes: A Companion to Braunwald's Heart Disease. [Internet]. 2014 [Consultado 30 de junio de 2020]. Disponible en: https://ebookcentral.proquest.com/lib/ qut/detail.action?docID $=1429681$

12. Coronado BE, Pope JH, Griffith JL, Beshansky JR, Selker HP. Clinical features, triage, and outcome of patients presenting to the ED with suspected acute coronary syndromes but without pain: A multicenter study. Am J Emerg Med 2004;22:568-74.

13. Canto JG. Prevalence, clinical characteristics, and mortality among patients with myocardial infarction presenting without chest pain. JAMA 2000;283:3223-9.

14. Giugliano RP, Cannon CP, Braunwald E. Non-ST-segment elevation acute coronary syndrome (non-ST-segment elevation myocardial infarction and unstable angina). En: Jameson JL, Fauci AS, Kasper DL, Hauser SL, Longo DL, Loscalzo J, editores. Harrison's Principles of Internal Medicine, 20e [Internet]. New York, NY: McGraw-Hill Education; 2018 [Consultado 2 de julio de 2020]. Disponible en: accessmedicine.mhmedical.com/content.aspx?aid $=1155974833$

15. Shin YS, Ahn S, Kim Y-J, Ryoo SM, Sohn CH, Kim WY. Risk stratification of patients with chest pain or anginal equivalents in the emergency department. Intern Emerg Med 2020;15:319-26.

16. Greenslade JH, Cullen L, Parsonage W, Reid CM, Body R, Richards M, et al. Examining the signs and symptoms experienced by individuals with suspected acute coronary syndrome in the Asia-Pacific region: A prospective observational study. Ann Emerg Med 2012;60:777-85.

17. Beltrán FM. Dolor torácico en el servicio de urgencias: "un reto por enfrentar". Rev Colomb Cardiol 2003;10(8):10.

18. Kawamoto KR, Davis MB, Duvernoy CS. Acute coronary syndromes: Differences in men and women. Curr Atheroscler Rep 2016;18:73.

19. Patel H, Rosengren A, Ekman I. Symptoms in acute coronary syndromes: Does sex make a difference? Am Heart J 2004;148:27-33.

20. Varghese T, Wenger NK. Non-ST elevation acute coronary syndrome in women and the elderly: Recent updates and stones still left unturned. F1000Res 2018;7: [Consultado 22 de septiembre de 2020];7:1865.

21. De Von HA, Mirzaei S, ZègreaHemsey J. Typical and atypical symptoms of acute coronary syndrome: Time to retire the terms? J Am Heart Assoc 2020;9:e015539.

22. Foussas SG. Acute coronary syndromes and diabetes mellitus. Hellenic J Cardiol 2016;57:375-77.

23. Franklin K, Goldberg RJ, Spencer F. Implications of diabetes in patients with acute coronary syndromes: The global registry of acute coronary events. Arch Intern Med 2004;164:1457-63.

24. De Von HA, Penckofer S, Larimer K. The association of diabetes and older age with the absence of chest pain during acute coronary syndromes. West J Nurs Res 2008;30:130-44. 
25. Krishnaswami A, Hardison R, Nesto RW, Sobel B. Presentation in patients with angiographically documented coronary artery disease and Type II diabetes mellitus (from the BARI 2D Clinical Trial). Am J Cardiol 2012;109:36-41.

26. Trichon BH, Roe MT. Acute coronary syndromes and diabetes mellitus. Diab Vasc Dis Res 2004;1:23-32.

27. Gillis NK, Arslanian-Engoren C, Struble LM. Acute coronary syndromes in older adults: A review of literature. J Emerg Nurs 2014;40:270-5.

28. Veerasamy M, Edwards R, Ford G, Kirkwood T, Newton J, Jones D, et al. Acute coronary syndrome among older patients: A review. Cardiol Rev 2015;23:26-32.

29. Alexander KP, Newby LK, Cannon CP, Armstrong PW, Gibler WB, Rich MW, et al. Acute coronary care in the elderly, Part I: Non-ST-segment-elevation acute coronary syndromes: A scientific statement for healthcare professionals from the American Heart Association Council on Clinical Cardiology: In collaboration with the Society of Geriatric Cardiology. Circulation 2007;115:2549-69.

30. Sheifer SE, Manolio TA, Gersh BJ. Unrecognized myocardial infarction. Ann Intern Med 2001;135:801.

31. Gevigney G, Ecochard R, Rabilloud M, Gaillard S, Cheneau E, Ducreux C, et al. Worsening of heart failure during hospital course of an unselected cohort of 2507 patients with myocardial infarction is a factor of poor prognosis: The PRIMA study. Eur J Heart Fail 2001;3:233-41.

32. Ponikowski P, Jankowska EA. Patogenia y presentación clínica de la insuficiencia cardiaca aguda. Rev Esp Cardiol 2015;68:331-7.

33. Nieminen MS, Brutsaert D, Dickstein K, Drexler H, Follath F, Harjola V-P, et al. EuroHeart Failure Survey II (EHFS II): A survey on hospitalized acute heart failure patients: description of population. Eur Heart J 2006;27:2725-36.

34. Harjola V, Parissis J, Bauersachs J, BrunnerDLa Rocca $H$, Bueno $H$, Čelutkienè J, et al. Acute coronary syndromes and acute heart failure: A diagnostic dilemma and highपrisk combination. A statement from the Acute Heart Failure Committee of the Heart Failure Association of the European Society of Cardiology. Eur J Heart Fail 2020;22:1298-314.

35. Boehme AK, Esenwa C, Elkind MSV. Stroke risk factors, genetics, and prevention. Circ Res 2017;120:472-95.

36. Simons M, Alpert JS, Wilson PW, Breall JA. Risk factors for adverse outcomes after non-ST elevation acute coronary syndromes [internet]. UpToDate 2019. [Consultado 2 de julio de 2020]. Disponible en: https://www.uptodate.com/contents/risk-factors-for-adverse-outcomes-afternon-st-elevation-acute-coronary-syndromes

37. Roe MT, Chen AY, Thomas L, Wang TY, Alexander KP, Hammill BG, et al. Predicting long-term mortality in older patients after non-ST-segment elevation myocardial infarction: The CRUSADE long-term mortality model and risk score. Am Heart J 2011;162:875-883.e1.

38. Virani SS, Alonso A, Benjamin EJ, Bittencourt MS, Callaway CW, Carson AP, et al. Heart disease and stroke statistics-2020 update: A report from the American Heart Association. Circulation 2020;141:e139-e596.

39. Collet J-P, Thiele H, Barbato E, Barthélémy O, Bauersachs J, Bhatt DL, et al. 2020 ESC Guidelines for the management of acute coronary syndromes in patients presenting without persistent ST-segment elevation. Eur Heart J 2020;14:1289-367.

40. Sun BC, Laurie A, Fu R, Ferencik M, Shapiro M, Lindsell CJ, et al. Comparison of the HEART and TIMI risk scores for suspected acute coronary syndrome in the emergency department. Crit Pathw Cardiol 2016;15:1-5.

41. Six AJ, Backus BE, Kelder JC. Chest pain in the emergency room: Value of the HEART score. Neth Heart J 2008;16:191-6.

42. Backus BE, Six AJ, Kelder JC, Bosschaert MAR, Mast EG, Mosterd A, et al. A prospective validation of the HEART score for chest pain patients at the emergency department. Int J Cardiol 2013;168:2153-8. 